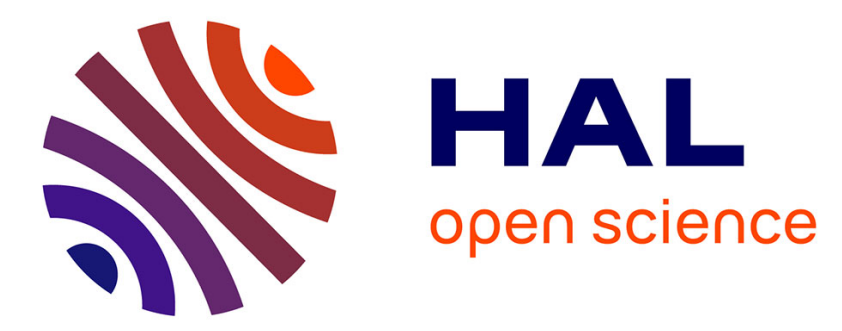

\title{
Some remarks on Smith predictors A geometric point of view
}

Irinel-Constantin Morarescu, Silviu-Iulian Niculescu, Keqin Gu

\section{To cite this version:}

Irinel-Constantin Morarescu, Silviu-Iulian Niculescu, Keqin Gu. Some remarks on Smith predictors A geometric point of view. 6th IFAC Workshop on Time Delay Systems, TDS 2006, Jul 2006, L'Aquila, Italy. pp.264-269. hal-02294066

\section{HAL Id: hal-02294066 \\ https://hal.science/hal-02294066}

Submitted on 24 Sep 2019

HAL is a multi-disciplinary open access archive for the deposit and dissemination of scientific research documents, whether they are published or not. The documents may come from teaching and research institutions in France or abroad, or from public or private research centers.
L'archive ouverte pluridisciplinaire HAL, est destinée au dépôt et à la diffusion de documents scientifiques de niveau recherche, publiés ou non, émanant des établissements d'enseignement et de recherche français ou étrangers, des laboratoires publics ou privés. 


\title{
SOME REMARKS ON SMITH PREDICTORS: A GEOMETRIC POINT OF VIEW
}

\author{
Constantin-Irinel Morărescu ${ }^{1}$ Silviu-Iulian Niculescu ${ }^{2}$ \\ Keqin $\mathbf{G u}^{3}$
}

\begin{abstract}
In this paper we develop a method to obtain the stability crossing curves of a Smith Predictor control scheme. More explicitly, we compute the crossing set, which consists of all frequencies corresponding to all points on the stability crossing curve, and we give their complete classification. Furthermore, the directions in which the zeros cross the imaginary axis are explicitly expressed.
\end{abstract}

Keywords: Smith Predictor, Delay uncertainty, Stability

\section{INTRODUCTION}

The stability and control of time-delay systems are subject of recurring interest since the delay is inherently present in various applications, from signal propagation in networks to population dynamics (see, for instance, [7, 2] for further references, and examples).

The aim of this paper is to present some new interpretations of the Smith predictors ([10]) subject to delay uncertainty. This problem was largely treated in the literature starting with the 80s (see, for instance, [8], [12], [11], [9], and the references therein), and it is reconsidered recently ([6]), mainly to the increasing interest of using such a methodology in applications,

\footnotetext{
1 HeuDiaSyC (UMR CNRS 6599), Université de Technologie de Compiègne, Centre de Recherche de Royallieu, BP 20529, 60205, Compiègne, France. E-mail: cmorares@hds . utc. fr; The author is also with the Department of Mathematics, University "Politehnica" of Bucharest, Romania. The work of C.-I. MORĂRESCU was (partially) supported through a E. C. Marie Curie Fellowship and in the framework of the CTS, HPMT-CT-2001-00278

2 HeuDiaSyC (UMR CNRS 6599), Université de Technologie de Compiègne, Centre de Recherche de Royallieu, BP 20529, 60205, Compiègne, France. E-mail: niculescu@hds.utc.fr; The work of S.-I. NICULESCU was partially funded by the CNRS-US Grant: "Delays in interconnected dynamical systems: Analysis, and applications" (2005-2007).

3 Department of Mechanical and Industrial Engineering, Southern Illinois University at Edwardsville, Edwardsville, Illinois 620261805, USA. E-mail: kgu@siue.edu. The work of K. GU was partially funded by the CNRS-US Grant: "Delays in interconnected dynamical systems: Analysis, and applications” (2005-2007).
}

like control of congestions in high-speed networks (see, e.g. [5]).

The approach considered in the paper makes use of some simple geometric idea (triangles inequality), inspired by ([3]) and devoted to the characterization of the stability crossing curves for general systems with two delays. The novelty of the results lies in a simple, and easy to follow classification of all the situations where uncertainty on the nominal delay value will induce instabilities in the corresponding schemes, and also considers, and gives the corresponding characterizations of some of the degenerate cases mentioned, but not discussed by [3]. Furthermore, the approach under consideration completes the algebraic characterizations in ([7], [6]).

\section{PROBLEM FORMULATION, AND PRELIMINARIES}

As discussed in [7], the analysis of the effects induced by the delay uncertainty of the Smith predictor on the stability of the corresponding closed-loop schemes reduce to the analysis of the following characteristic equation:

$$
P(s)+Q(s) \mathrm{e}^{-s \tau_{1}}-Q(s) \mathrm{e}^{-s\left(\tau_{1}+\delta\right)}=0,
$$

where $\tau_{1}$ represents the nominal delay value, and $P(s), Q(s)$ are appropriate polynomials defined by the plant free of delay, and by corresponding controller. 
Let $\mathcal{G}$ be the set of all pairs $(x, y) \in \mathbb{R}_{+}^{2}$ such that $x<y$. It is obvious that replacing $\tau_{2}=\tau_{1}+\delta$ and taking $\left(\tau_{1}, \tau_{2}\right) \in \mathcal{G}$ we can consider the following equivalent equation:

$D\left(s, \tau_{1}, \tau_{2}\right)=P(s)+Q(s) \mathrm{e}^{-s \tau_{1}}-Q(s) \mathrm{e}^{-s \tau_{2}}=0$.

More explicitly, we study the occurrence of any possible stability switch/reversal ${ }^{4}$ resulting by increasing the time delay $\tau_{1}$ or $\tau_{2}$. In other words, we explicitly study the change of number of zeros of (1) on $\mathbb{C}_{+}$as the delays $\left(\tau_{1}, \tau_{2}\right)$ vary on $\mathcal{G}$. Since the main objective of this study is to identify the regions of $\left(\tau_{1}, \tau_{2}\right)$ in $\mathcal{G}$ such that $D\left(s, \tau_{1}, \tau_{2}\right)$ is (asymptotically) stable, we will exclude some cases, and the following assumptions appear naturally:

Assumption 1. $\operatorname{deg}(Q) \leq \operatorname{deg}(P)$.

Assumption 2. $P(0) \neq 0$.

Assumption 3. P, $Q$ do not have common zeros.

Assumption 4. $\mathrm{P}$ and $\mathrm{Q}$ are such that:

$$
\lim _{s \rightarrow \infty}\left|\frac{Q(s)}{P(s)}\right|<\frac{1}{2} .
$$

Remark 1. If the system is of retarded type then the assumption 4 is automatically satisfied since its left hand is zero. For neutral systems, let $c=\lim _{s \rightarrow \infty} \frac{Q(s)}{P(s)}$. Then it is well known that the stability of the system (1) is possible only if the difference equation

$$
x(t)+c x\left(t-\tau_{1}\right)-c x\left(t-\tau_{2}\right)=0
$$

is exponentially stable. Assumption 4 guarantees the stability of (2).

\section{IDENTIFICATION OF THE CROSSING POINTS}

Let $\mathcal{T}$ denote the set of all points $\left(\tau_{1}, \tau_{2}\right) \in \mathcal{G}$ such that $D(s)$ has at least one zero on the imaginary axis. Any $\left(\tau_{1}, \tau_{2}\right) \in \mathcal{T}$ is known as a crossing point. The set $\mathcal{T}$, which is the collection of all crossing points, is known as the stability crossing curves. Let $\mathcal{T}_{\omega}$ denote the set of all $\left(\tau_{1}, \tau_{2}\right) \in \mathcal{G}$ such that $D(s)$ has at least one zero for $s=j \omega$. Let $\Omega$ the set of all $\omega$ for which there exists a pair $\left(\tau_{1}, \tau_{2}\right)$ such that $D\left(j \omega, \tau_{1}, \tau_{2}\right)=0$. We will refer to $\Omega$ as the crossing set. Obviously

$$
\mathcal{T}=\left\{\mathcal{T}_{\omega} \mid \omega \in \Omega\right\}
$$

Next, for the clarity of the presentation we will split our discussion in two parts. First we will consider only the case which satisfy the following non-degeneracy condition,

\footnotetext{
4 We are using the same terminology as in Cooke and Grossman [1], that is a root of the characteristic equation crossing the imaginary axis, when some parameter is varying.
}

Assumption 5.

$$
P(j \omega) \cdot Q(j \omega) \neq 0 \text { for all } \omega \in \Omega
$$

and then we will discuss what happens in other cases.

\subsection{Regular cases}

In the sequel, we consider

$$
h(s)=\frac{Q(s)}{P(s)}
$$

and

$$
H(s)=1+h(s) e^{-s \tau_{1}}-h(s) e^{-s \tau_{2}}
$$

For given $\tau_{1}$ and $\tau_{2}$, as long as assumption 5 is satisfied, $D(s)$ and $H(s)$ share all the zeros in a neighborhood of the imaginary axis. Therefore, we may obtain all the crossing points and direction of crossing using $H(s)=0$ instead of $D(s)=0$. We may also consider the three terms in $H(s)$ as three vectors in the complex plane, with magnitudes $1,|h(s)|$ and $|h(s)|$ respectively. So when we adjust the values of $\tau_{1}$ and $\tau_{2}$ in fact we adjust the directions of the vectors represented by the second and the third terms. Equation (4) means that if we put the first two vectors had to tail then we get the third vector. In other words they form an isosceles triangle and the existence condition reduces to: the sum of the equal sides exceed the other side. This allows us to give the following proposition.

Proposition 1. For some $\left(\tau_{1}, \tau_{2}\right) \in \mathcal{G}, H(s)$ has an imaginary zero $s=j \omega, \omega \neq 0$ if and only if

$$
|h(j \omega)| \geq \frac{1}{2} .
$$

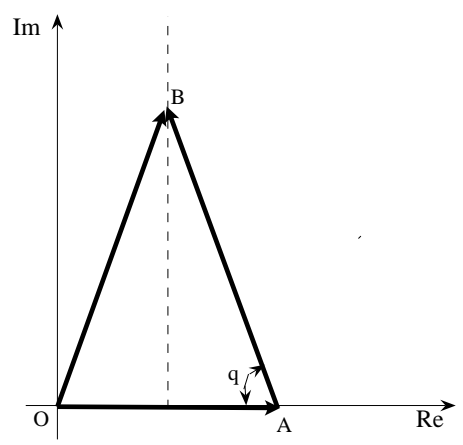

Fig. 1. Triangle formed by $1, h(s) e^{-s \tau_{1}}$ and $h(s) e^{-s \tau_{2}}$

Due to the symmetry and assumption 2 we only need to consider positive $\omega$. So $\Omega$ is the set of all $\omega>0$ which satisfy (5). Also, for a given $\omega \in \Omega$ we may find all the pairs $\left(\tau_{1}, \tau_{2}\right)$ satisfying $H(j \omega)=0$ as follows: 


$$
\begin{aligned}
\tau_{1}=\tau_{1}^{u \pm}(\omega) & =\frac{\angle h(j \omega)+(2 u-1) \pi \pm q}{\omega}, \\
u & =u_{0}^{ \pm}, u_{0}^{ \pm}+1, u_{0}^{ \pm}+2, \ldots \\
\tau_{2}=\tau_{2}^{v \pm}(\omega) & =\frac{\angle h(j \omega)+2 v \pi \mp q}{\omega}, \\
v & =v_{0}^{ \pm}, v_{0}^{ \pm}+1, v_{0}^{ \pm}+2, \ldots
\end{aligned}
$$

where $q \in[o, \pi]$ is the internal angle of triangle in Figure 1 which can be calculated by the cosine law as

$$
q(j \omega)=\cos ^{-1}\left(\frac{1}{2|h(\omega)|}\right)
$$

and $u=u_{0}^{+}, u=u_{0}^{-}, v=v_{0}^{+}, v=v_{0}^{-}$are the smallest integers (may depend on $\omega$ ) such that the corresponding values $\tau_{1}^{u_{0}^{+}+}, \tau_{1}^{u_{0}^{-}-}, \tau_{2}^{u_{0}^{+}+}, \tau_{2}^{u_{0}^{-}-}$are nonnegative and $\tau_{2}^{u_{0}^{+}+}>\tau_{1}^{u_{0}^{+}+}, \tau_{2}^{u_{0}^{-}-}>\tau_{1}^{u_{0}^{-}-}$. The position in Figure 1 corresponds to $\left(\tau_{1}^{u+}, \tau_{2}^{u+}\right)$ and the mirror image about the real axis corresponds to $\left(\tau_{1}^{u-}, \tau_{2}^{u-}\right)$. If we define $\mathcal{T}_{\omega, u, v}^{+}$and $\mathcal{T}_{\omega, u, v}^{-}$as the singletons $\left(\tau_{1}^{u+}(\omega), \tau_{2}^{u+}(\omega)\right)$ and $\left(\tau_{1}^{u-}(\omega), \tau_{2}^{u-}(\omega)\right)$ respectively, then we can characterize $\mathcal{T}_{\omega}$ as follows:

$\mathcal{T}_{\omega}=\left(\bigcup_{u \geq u_{0}^{+}, v \geq v_{0}^{+}} \mathcal{T}_{\omega, u, v}^{+}\right) \bigcup\left(\bigcup_{u \geq u_{0}^{-}, v \geq v_{0}^{-}} \mathcal{T}_{\omega, u, v}^{-}\right)$

Proposition 2. The crossing set $\Omega$ consists of a finite number of intervals of finite length including the cases which may violate assumption 5 .

Proof: First one can observe easily that the number of points in $\Omega$ violating (3) is finite. So, we only need to show that the set of all points satisfying (5) consists of a finite number of intervals of finite length. Because

$$
|h(j \omega)|=\frac{1}{2} \Leftrightarrow|P(j \omega)|=2|Q(j \omega)|
$$

is a polynomial equation of variable $\omega^{2}$ it has a finite number of positive solutions. Therefore the solution of (5) consists of a finite number of intervals. Due to assumption 4 , any sufficiently large $\omega$ violates (5). Therefore the lengths of all intervals are finite. In what follows we will denote these intervals as $\Omega_{1}, \Omega_{2}, \ldots, \Omega_{N}$ and without loss of generality we may suppose that the intervals are ordered such that for any $\omega_{1} \in \Omega_{k_{1}}, \omega_{2} \in \Omega_{k_{2}}, k_{1}<k_{2}$ we have $\omega_{1}<\omega_{2}$.

Remark 2. If (5) is satisfied for $\omega=0$ and sufficiently small positive value of $\omega$ then we will take 0 as the left end of $\Omega_{1}$. Considering $\omega_{1}^{r}$ the right end of $\Omega_{1}$, according to assumption 2 we get $\Omega_{1}=\left(0, \omega_{1}^{r}\right]$, so $0 \notin \Omega$.

Remark 3. From geometrical point of view an end point $\omega^{*}$ with $\left|P\left(j \omega^{*}\right)\right|=2\left|Q\left(j \omega^{*}\right)\right|$, corresponds to the limit case where internal angle $q$ of the triangle is 0 . In this case we obtain $\overrightarrow{O B}=-\overrightarrow{A B}$ on the real axis.

We will not restrict $\angle h(j \omega)$ to be within the $2 \pi$ range but make it a continuous function of $\omega$ within each
$\Omega_{k}$. Thus, for each fixed $u, v$ and $k,(6)$ and (7) are continuous curves denoted as $\mathcal{T}_{u, v}^{k+}$ respectively $\mathcal{T}_{u, v}^{k-}$. We should keep in mind that, for some $u, v$ and $k$, part or entire curve $\mathcal{T}_{u, v}^{k+}$ (respectively $\mathcal{T}_{u, v}^{k-}$ ) may be outside of the range $\mathcal{G}$, and therefore, may not be physically meaningful. The collection of all the points in $\mathcal{T}$ corresponding to $\Omega_{k}$ may be expressed as

$$
\begin{aligned}
\mathcal{T}^{k} & =\bigcup_{u=-\infty}^{\infty} \bigcup_{v=-\infty}^{\infty}\left[\left(\mathcal{T}_{u, v}^{k+} \cup \mathcal{T}_{u, v}^{k-}\right) \cap \mathcal{G}\right]=\bigcup_{\omega \in \Omega_{k}} \mathcal{T}_{\omega} \\
\mathcal{T} & =\bigcup_{k=1}^{N} \mathcal{T}^{k}
\end{aligned}
$$

Our previous discussions allow us to say that the ends of $\Omega_{k}$ must be in one of the following situation:

Type 1. It satisfies the equation $|h(x)|=\frac{1}{2}$.

Type 2. It equals 0 .

If one end of $\Omega_{k}$ is of type 1 then $q=0$ and $\mathcal{T}_{u, v}^{k+}$ is connected with $\mathcal{T}_{u, v}^{k-}$ at this end. So, if both ends of $\Omega_{k}$ are of type 1 we get $\mathcal{T}^{k}$ is a series of closed curves.

Obviously just the left end of $\Omega_{1}$ can be 0 . In this case, as $\omega \rightarrow 0$, both $\tau_{1}$ and $\tau_{2}$ approach $\infty$. In fact $\mathcal{T}_{u, v}^{1+}$ and $\mathcal{T}_{u, v}^{1-}$ approach $\infty$ with asymptotes passing through the points $(\hat{h} \pm \hat{q}, \hat{h} \mp \hat{q})$ with slopes of

$$
m_{u, v}^{ \pm}=\frac{\tau_{2}^{v \pm}}{\tau_{1}^{u \pm}}=\frac{\angle h(0)+2 v \pi \mp q(0)}{\angle h(0)+(2 u-1) \pi \pm q(0)},
$$

where $q(0)$ is evaluated using (8) and

$$
\hat{h}=\left.\frac{\mathrm{d}}{\mathrm{d} \omega}[\angle h(j \omega)]\right|_{\omega=0}, \hat{q}=\left.\frac{\mathrm{d}}{\mathrm{d} \omega}[q(j \omega)]\right|_{\omega=0} .
$$

In the sequel we will say that an interval is of type 11 if both his end are of type 1 , and $\Omega_{1}$ is of type 21 if his left end is 0 . Therefore, the crossing set $\Omega$ consists in a finite number of intervals of type 11 , and eventually the first interval is of type 21 . It is obvious that $\mathcal{T}^{k}$ consists in a series of curves belonging to one of the following categories:

A) A series of closed curves $\left(\Omega_{k}\right.$ is of type 11)

B) A series of open ended curves with both ends approaching $\infty\left(\Omega_{k}\right.$ is of type 21$)$

We continue this section with some illustrative examples regarding the above characterization.

Example 1. (type 11) Consider a system with

$$
h(s)=\frac{4 s+1}{4\left(s^{2}+s+1\right)}
$$

Figure 2 (up) plots $2|h(j \omega)|$ against $\omega$. The crossing set can be easily identified from this figure, it contains one interval $\Omega_{1}=[0.39,2,21]$

As an illustration of a series of closed curves we examine $\mathcal{T}^{k}$ corresponding to $\Omega_{k}$ of type 11 . In this case, for a given $u$ and $v$ such that $\tau_{2}^{u \pm}>\tau_{1}^{u \pm}>0$, 

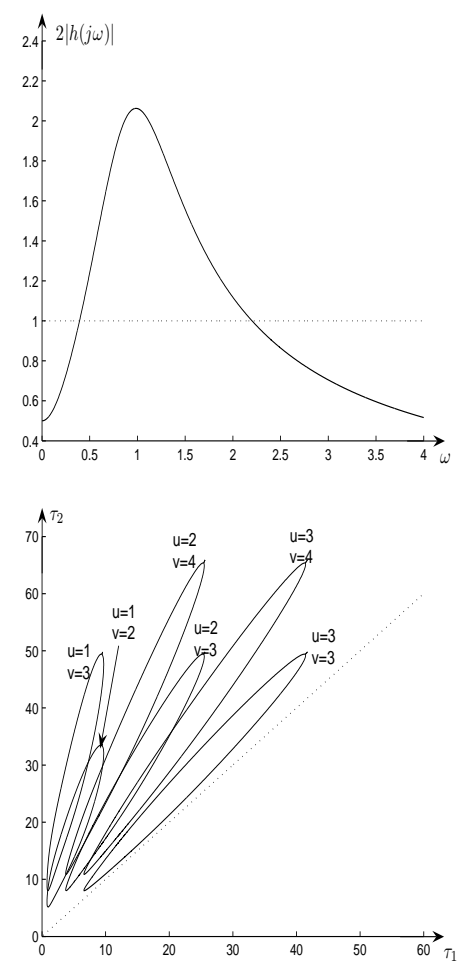

Fig. 2. The crossing set for the system (10) can be identified up and some crossing curves of this system are plotted down

we get $\mathcal{T}_{u, v}^{k+}$ and $\mathcal{T}_{u, v}^{k-}$ are connected on the both ends to form a closed curve. As $u$ and $v$ vary, we obtain a series of deformed versions of such closed curves situated above the first bisector. A suggestive image of a series of closed curves is given in Figure 2 (down) which show $\mathcal{T}$ of the system described in (10).

Example 2. (type 21) Consider a system with

$$
h(s)=\frac{s+\sqrt{2}}{2 s^{3}+s^{2}+8 s+1}
$$

Figure 3 (up) plots $|h(j \omega)|$ against $\omega$. The crossing set $\Omega$ can be easily identified from the Figure 3 , it contains two intervals: $\Omega_{1}=(0,0.364]$ of type 21 , and $\Omega_{2}=[1.673,2.198]$ of type 11 .

In the sequel, we consider $\Omega_{k}=\left[\omega_{k}^{l}, \omega_{k}^{r}\right]$. Obviously, the interval $\Omega_{1}$ is open to the left if its left end is 0 . To illustrate the case of open ended curves we consider $\mathcal{T}^{1}$ corresponding to $\Omega_{1}$ of type 21 . In this case $\Omega_{1}=$ $\left(0, \omega_{1}^{r}\right]$ and for a given $u$ and $v, \mathcal{T}_{u, v}^{1+}$ and $\mathcal{T}_{u, v}^{1-}$ are connected at $\omega_{1}^{r}$. The other end of $\mathcal{T}_{u, v}^{1-}$ extends to infinity with asymptotes passing through the points $(\hat{h}-\hat{q}, \hat{h}+\hat{q})$ with the slope $m_{u, v}^{-}$and, the other end of $\mathcal{T}_{u, v}^{1+}$ extends to infinity with asymptotes passing through the points $(\hat{h}+\hat{q}, \hat{h}-\hat{q})$ with the slope $m_{u, v}^{+}$. Again, as $u$ and $v$ vary, we obtain a series of deformed versions of such open ended curves situated above the first bisector. Evidently, the slope is changing for different $u$ and $v$. We can see a series of open ended curves in Figure 3 (right).
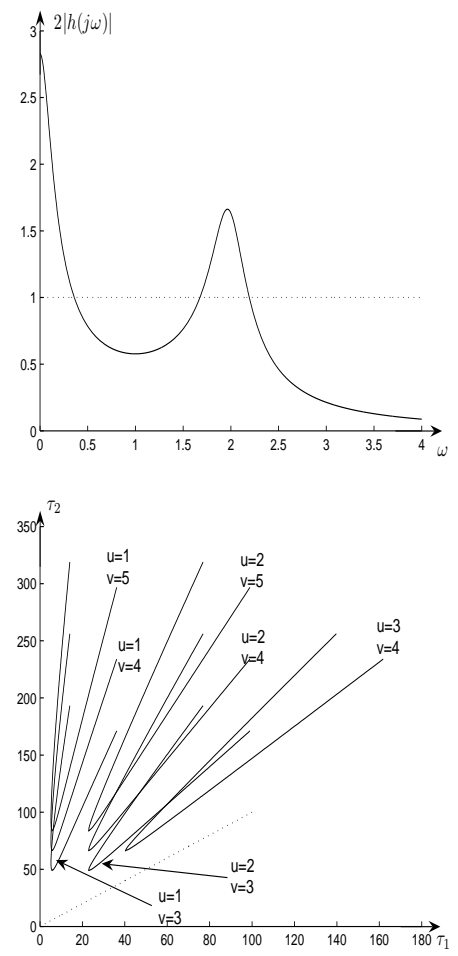

Fig. 3. The crossing set for the system (12) can be identified up and some crossing curves of this system are plotted down

\subsection{Degenerate cases}

In the sequel, we consider the cases violating assumption 5. Obviously, the interesting case is $P(j \omega)=0$ has at least one positive solution. We can easily state the following:

Remark 4. . For $\omega^{*} \neq 0$ satisfying $Q\left(j \omega^{*}\right)=0$ it is clear that increasing of $\tau_{1}$ and/or $\tau_{2}$ has no effect w.r.t. stability of the system. We note also that $h\left(j \omega^{*}\right)=0$ imply that $h(j \omega)<\frac{1}{2}$, for all $\omega$ in a neighborhood of $\omega^{*}$.

Next, we assume that $P\left(j \omega^{*}\right)=0$ for $\omega^{*} \neq 0$. Using assumption 3 we get $Q\left(j \omega^{*}\right) \neq 0$ and therefore $\lim _{\omega \rightarrow \omega^{*}}|h(j \omega)|=\infty$. So that, $|h(j \omega)|>\frac{1}{2}$ for all $\omega$ in a neighborhood of $\omega^{*}$. This mean that $\Omega$ contains one interval of type $\left[\omega^{l}, \omega^{*}\right]$ and one of type $\left[\omega^{*}, \omega^{r}\right]$. It is clear that the first interval is open to the left if $\omega^{l}=0$.

Proposition 3. For $\omega^{*} \neq 0$ satisfying $P\left(j \omega^{*}\right)=0$ we get $\mathcal{T}_{\omega^{*}}$ consists of the solutions of

$$
\omega^{*} \tau_{2}=\omega^{*} \tau_{1}+2 m \pi, m \in \mathbb{Z} \text { in } \mathcal{G} \text {. }
$$

and

$$
\begin{aligned}
\lim _{\omega \rightarrow \omega^{*}} q(j \omega) & =\frac{\pi}{2} ; \\
\lim _{\omega \rightarrow \omega^{*}} \tau_{1}(\omega) & =\frac{2 \angle Q\left(j \omega^{*}\right)+(4 u-2 \pm 1) \pi}{2 \omega^{*}} ; \\
\lim _{\omega \rightarrow \omega^{*}} \tau_{2}(\omega) & =\frac{2 \angle Q\left(j \omega^{*}\right)+(4 v \mp 1) \pi}{2 \omega^{*}} .
\end{aligned}
$$


Proof. Straightforward computations.

Remark 5. 1) $\mathcal{T}_{\omega^{*}}$ consists of an infinite number of straight lines of slope 1 of equal distance.

2) $\lim _{\omega \rightarrow \omega^{*}} \tau_{2}(\omega)-\lim _{\omega \rightarrow \omega^{*}} \tau_{1}(\omega)=2 m \pi, m \in \mathbb{Z}$

Let $\Omega_{k}=\left[\omega^{l}, \omega^{*}\right]$ and $\Omega_{k+1}=\left[\omega^{*}, \omega^{r}\right]$. In this case, using proposition 3 we get $\mathcal{T}_{u, v}^{k+}$ is connected with $\mathcal{T}_{u, v}^{(k+1)+}$ and $\mathcal{T}_{u, v}^{k-}$ is connected with $\mathcal{T}_{u, v}^{(k+1)-}$ at the end corresponding to $\omega^{*}$. Using (9) and remark 5 we obtain that each crossing curve in $\mathcal{T}_{u, v}^{k \pm}$ consist of an union of one straight line of slope 1 and the curve corresponding to $\Omega_{k} \backslash\left\{\omega^{*}\right\}$. From Remark 5 we deduce that one end of the curve corresponding to $\Omega_{k} \backslash\left\{\omega^{*}\right\}$ is on the line in $\mathcal{T}_{\omega^{*}}$ which correspond to the pair $(u, v)$. In the following if $P\left(j \omega^{*}\right)=0$ we will say that $\omega^{*}$ is an end point of type 0 .

Example 3. (type 20 and 01) Consider a system with

$$
h(s)=\frac{s+2}{s^{2}+2}
$$

Figure 3 (up) plots $\frac{1}{|h(j \omega)|}$ against $\omega$. The crossing set $\Omega$ contains two intervals: $\Omega_{1}=(0, \sqrt{2}]$ of type 20 , and $\Omega_{2}=[\sqrt{2}, 3.046]$ of type 01 . Figure 4 (down) plots $\mathcal{T}_{3,4}^{2 \pm}$ which are two curves of type 01 .
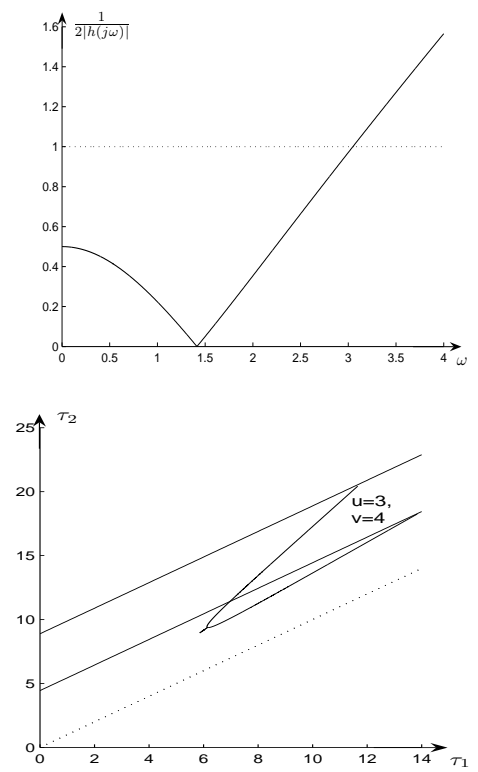

Fig. 4. The crossing set for the above system can be identified up and $\mathcal{T}_{3,4}^{2 \pm}$ of this system are plotted down

\section{CROSSING CURVES, CHARACTERISTIC ROOTS BEHAVIOR}

\subsection{Tangent and Smoothness}

For a given $k$ we will discuss the smoothness of the curves in $\mathcal{T}^{k}$ and thus $\mathcal{T}=\bigcup_{k=1}^{N} \mathcal{T}^{k}$. In this part we use an approach based on the implicit function theorem. For this purpose we consider $\tau_{1}$ and $\tau_{2}$ as implicit functions of $s=j \omega$ defined by (1).

For a given $k$, as $s$ moves along the imaginary axis within $\Omega_{k},\left(\tau_{1}, \tau_{2}\right)=\left(\tau_{1}^{u \pm}(\omega), \tau_{2}^{v \pm}(\omega)\right)$ moves along $\mathcal{T}^{k}$. For a given $\omega \in \Omega_{k}$, let

$$
\begin{aligned}
R_{0} & =\operatorname{Re}\left(\frac{j}{s} \frac{\partial D\left(s, \tau_{1}, \tau_{2}\right)}{\partial s}\right)_{s=j \omega} \\
& =\frac{1}{\omega} \operatorname{Re}\left\{\left[h^{\prime}(j \omega)-\tau_{1} h(j \omega)\right] e^{-j \omega \tau_{1}}\right. \\
& \left.+\left[\tau_{2} h(j \omega)-h^{\prime}(j \omega)\right] e^{-j \omega \tau_{2}}\right\}, \\
I_{0} & =\operatorname{Im}\left(\frac{j}{s} \frac{\partial D\left(s, \tau_{1}, \tau_{2}\right)}{\partial s}\right)_{s=j \omega} \\
& =\frac{1}{\omega} \operatorname{Im}\left\{\left[h^{\prime}(j \omega)-\tau_{1} h(j \omega)\right] e^{-j \omega \tau_{1}}\right. \\
& \left.+\left[\tau_{2} h(j \omega)-h^{\prime}(j \omega)\right] e^{-j \omega \tau_{2}}\right\}
\end{aligned}
$$

and

$$
\begin{aligned}
R_{l} & =\operatorname{Re}\left(\frac{1}{s} \frac{\partial D\left(s, \tau_{1}, \tau_{2}\right)}{\partial \tau_{l}}\right)_{s=j \omega}=(-1)^{l-1} \operatorname{Re}\left(h(j \omega) e^{-j \omega \tau_{l}}\right), \\
I_{l} & =\operatorname{Im}\left(\frac{1}{s} \frac{\partial D\left(s, \tau_{1}, \tau_{2}\right)}{\partial \tau_{l}}\right)_{s=j \omega}=(-1)^{l-1} \operatorname{Im}\left(h(j \omega) e^{-j \omega \tau_{l}}\right),
\end{aligned}
$$

for $1=1,2$. Then, since $D\left(s, \tau_{1}, \tau_{2}\right)$ is an analytic function of $s, \tau_{1}$ and $\tau_{2}$, the implicit function theorem indicates that the tangent of $\mathcal{T}^{k}$ can be expressed as

$$
\left(\begin{array}{c}
\frac{\mathrm{d} T}{\mathrm{~d} \omega} \\
\frac{\mathrm{d} \tau}{\mathrm{d} \omega}
\end{array}\right)=\frac{1}{R_{1} I_{2}-R_{2} I_{1}}\left(\begin{array}{c}
R_{0} I_{2}-I_{0} R_{2} \\
I_{0} R_{1}-R_{0} I_{1}
\end{array}\right),
$$

provided that

$$
R_{1} I_{2}-R_{2} I_{1} \neq 0 .
$$

It follows that $\mathcal{T}_{k}$ is smooth everywhere except possibly at the points where either (14) is not satisfied, or when

$$
\frac{\mathrm{d} T}{\mathrm{~d} \omega}=\frac{\mathrm{d} \tau}{\mathrm{d} \omega}=0
$$

From the above discussions, we can conclude:

Proposition 4. The curve $T^{k}$ is smooth everywhere except possibly at the points corresponding to $s=j \omega$ a multiple solution of (1).

\subsection{Direction of crossing}

Next, we will discuss the direction in which the solutions of (1) cross the imaginary axis as $\left(\tau_{1}, \tau_{2}\right)$ deviates from the curve $\mathcal{T}^{k}$. We will call the direction of 
the curve that corresponds to increasing $\omega$ the positive direction. We will also call the region on the left hand side as we head in the positive direction of the curve the region on the left.

To establish the direction of crossing we need to consider $\tau_{1}$ and $\tau_{2}$ as functions of $s=\sigma+j \omega$, i.e., functions of two real variables $\sigma$ and $\omega$, and partial derivative notation needs to be adopted. Since the tangent of $\mathcal{T}^{k}$ along the positive direction is $\left(\frac{\partial \tau_{1}}{\partial \omega}, \frac{\partial \tau_{2}}{\partial \omega}\right)$, the normal to $\mathcal{T}^{k}$ pointing to the left hand side of positive direction is $\left(-\frac{\partial \tau_{2}}{\partial \omega}, \frac{\partial \tau_{1}}{\partial \omega}\right)$. Corresponding to a pair of complex conjugate solutions of (1) crossing the imaginary axis along the horizontal direction, $\left(\tau_{1}, \tau_{2}\right)$ moves along the direction $\left(\frac{\partial \tau_{1}}{\partial \sigma}, \frac{\partial \tau_{2}}{\partial \sigma}\right)$. So, if a pair of complex conjugate solutions of (1) cross the imaginary axis to the right half plane, then,

$$
\left(\frac{\partial \tau_{1}}{\partial \omega} \frac{\partial \tau_{2}}{\partial \sigma}-\frac{\partial \tau_{2}}{\partial \omega} \frac{\partial \tau_{1}}{\partial \sigma}\right)_{s=j \omega}>0
$$

i.e. the region on the left of $\mathcal{T}^{k}$ gains two solutions on the right half plane. If the inequality (16) is reversed then the region on the left of $\mathcal{T}^{k}$ loses has two right half plane solutions. Similar to (13) we can express

$$
\left(\begin{array}{c}
\frac{\mathrm{d} \tau_{1}}{\mathrm{~d} \sigma} \\
\frac{\mathrm{d} \tau_{2}}{\mathrm{~d} \sigma}
\end{array}\right)_{s=j \omega}=\frac{1}{R_{1} I_{2}-R_{2} I_{1}}\left(\begin{array}{c}
R_{0} R_{2}+I_{0} I_{2} \\
-R_{0} R_{1}-I_{0} I_{1}
\end{array}\right) .
$$

Using this, we arrive to the following:

Proposition 5. Let $\omega \in\left(\omega_{k}^{l}, \omega_{k}^{r}\right)$ and $\left(\tau_{1}, \tau_{2}\right) \in$ $T^{k}$ such that $j \omega$ is a simple solution of (1) and $D\left(j \omega^{\prime}, \tau_{1}, \tau_{2}\right) \neq 0, \forall \omega^{\prime}>0, \omega^{\prime} \neq \omega$ (i.e. $\left(\tau_{1}, \tau_{2}\right)$ is not an intersection point of two curves or different sections of a single curve of $\mathcal{T}$ ). Then a pair of solutions of (1) cross the imaginary axis to the right, through $s= \pm j \omega$ if $R_{2} I_{1}-R_{1} I_{2}>0$. The crossing is to the left if the inequality is reversed.

\section{ILLUSTRATIVE EXAMPLES}

In this paragraph we reconsider one example already treated in the literature [6]. Consider:

$P(s)=\left(k_{1} k_{2}+1\right) s+\left(a+k_{1}\right), \quad Q(s)=k_{1}\left(k_{2} s+1\right)$.

[6] assume $a>0$ and $\left(a+k_{1}\right) /\left(k_{1} k_{2}+1\right)>0$, which guarantees internal stability of the closed-loop system. The so-called "practical stability" criterion is given by assumption 4 which simply states $\left|\frac{k_{1} k_{2}}{1+k_{1} k_{2}}\right|<$ $\frac{1}{2} \Leftrightarrow-1 / 3<k_{1} k_{2}<1$. For $a=1, k_{1}=2, k_{2}=$ $1 / 4$ we get $\Omega=(0,2.37]$, and, in conclusion, $\Omega$ consists of one interval of type 21 . More precisely, we obtain a series of open ended curves with both ends approaching infinity, conclusion which is similar to the one in [6], but derived using a different (algebraic) approach.

\section{CONCLUDING REMARKS}

This paper focuses on the stability crossing curves for a class of delay systems controlled by a Smith predictor, subject to uncertainty in the delay. More precisely, the particular form of the closed-loop system allows an easy derivation of the stability crossing curves (crossing set characterization, direction of crossing, smoothness). Regular, and degenerate cases are both treated. Various examples complete the presentation.

\section{REFERENCES}

[1] Cooke, K. L. and Grossman, Z.: Discrete delay, distributed delay and stability switches. J. Math. Anal. Appl. 86 (1982) 592-627.

[2] Gu, K., Kharitonov, V.L. and Chen, J. (2003). Stability and robust stability of time-delay systems. (Birkhauser: Boston).

[3] Gu, K., Niculescu, S.-I., and Chen, J. (2005). On stability of crossing curves for general systems with two delays, J. Math. Anal. Appl. 311, 231253.

[4] Hale, J. K. and Huang, W. (1993). Global geometry of the stable regions for two delay differential equations. J. Math. Anal. Appl., 178, 344-362.

[5] Mascolo, S. (1997). Smith's principle for congestion control in high-speed data networks. Proc. 37th IEEE Conf. Dec. Contr., San Diego, CA.

[6] Michiels, W., and Niculescu, S.-I. (2003). On the delay sensitivity of Smith Predictors, Int.J. of Systems Science, 34, 543-551.

[7] Niculescu, S.-I. (2001). Delay effects on stability. A robust control approach (Springer: Heidelberg, LNCIS, vol. 269).

[8] Palmor, Z. J. (1980). Stability properties of Smith dead-time compensator controllers. in Int. J. Contr. 32, 937-949.

[9] Palmor, Z. J. (1996). Time-delay compensation - Smith predictor and its modifications, in The Control Handbook (W. S. Levine, Eds.), CRC Press, 224-237.

[10] Smith, O. J. M. (1959). A controller to overcome dead time. Instrument Society of America Journal (ISA) 6, 28-33.

[11] Santacesaria, C. and Scattolini, R.: Easy tuning of Smith predictor in presence of delay uncertainty. in Automatica 29 (1993) 1595-1597.

[12] Yamanaka, K. and Shimemura, E. (1987). Effects of mismatched Smith controller on stability in systems with time-delay. in Automatica 23, 787-791. 